- Crisp, A. H. \& CAllender, J. (1992) Psychiatric diagnoses in recovered and non-recovered anorectics 22 years after onset of illness - pilot study. Comprehensive Psychiatry, 33, 123-127.

Eating Disorders Clinic

L. K. GeORGe Hsu

University of Pittsburgh

3811 O'Hara Street

Pittsburgh, PA 15213-2593

\section{Psychiatric symptoms in cannabis users}

SIR: Thomas (Journal, August 1993, 163, 141-149) reviewed the evidence for 'cannabis psychosis' being valid as a diagnostic entity. We have investigated this issue empirically in a consecutive series of psychotic admissions which has been described elsewhere (Jones et al, Journal, January 1993, 162, 65-71). The Present State Examination (PSE) was used to assess the psychopathology of 23 acute psychotic admissions who were cannabis positive on urinary screening, and 46 matched drug-free psychotic controls. Cases and controls were indistinguishable in terms of the prevalence of PSE syndromes. Moreover, the groups were also similar with respect to DSM-III-R diagnoses, ethnicity and socio-economic class, differing only, as expected, in terms of their histories of substance use.

We also examined the morbid risk of psychiatric illness in first-degree relatives, using maternal interviews and RDC-FH criteria, and Weinberg's shorter method of age correction. Cannabis positive cases had a significantly higher familial morbid risk of schizophrenia than the controls $(7.1 \%$ v. $0.7 \%$; odds ratio $=10.2 ; 95 \% \mathrm{CI} 1.12-234, P=0.02$ ), but similar rates for other conditions.

We agree with the author's conclusion that there is little evidence to support the concept of a 'cannabis psychosis'. Furthermore, we propose that psychosis occurring in the context of cannabis use may be particularly likely in those with a genetic predisposition to psychotic illness; cannabis use may trigger a 'functional' psychosis in those with a psychotic diathesis, rather than producing a specific 'cannabis psychosis' de novo.

PHILIP MCGUIRE PETER JONES

Institute of Psychiatry

De Crespigny Park

Denmark Hill

London SE5 8AF

Fluoxetine - induced mania in a patient with poststroke depression

SIR: Although mania or hypomania are well recognised side-effects of fluoxetine in depressed patients with 'functional' unipolar depression (Settle \& Settle, 1984) or bipolar disorder (Lebegue, 1987), cases of mania induced by fluoxetine in patients with 'organic' mood disorders have not been reported. The following case report describes a patient with poststroke depression (PSD), who developed mania three weeks after starting low doses of fluoxetine.

Case report. A 63-year-old right-handed woman suffered a sudden left hemiparesis and dysarthria in association with an ischemic infarction that involved the right corona radiata. Two months after the stroke, the patient became moderately depressed. Clinical features of depression included sadness, anhedonia, social withdrawal, inappropriate guilt, recurrent thoughts of death, and initial insomnia. On the 17-item Hamilton Depression Rating Scale her score was 16 points. She had no personal or family history of bipolar disorder, mania or any other psychiatric disorder.

The patient was started on a regimen of fluoxetine $(20 \mathrm{mg} /$ day $)$, and over the following days her depressive symptoms became less severe. Three weeks after starting fluoxetine, she became euphoric and markedly intrusive and had pressured speech, as well as flight of ideas and increased activity and libido. She also exhibited inflated self-esteem, impulsive spending, and diminished need for sleep. She met DSM-III-R criteria for organic affective disorder, manic type, and on the Beck Mania Scale her score was 19 points (definite mania). Fluoxetine was discontinued. Lithium carbonate $(800 \mathrm{mg} /$ day $)$ was started, and the patient experienced a rapid recovery.

Nortriptyline, trazodone and electroconvulsive therapy have all been reported to be effective and safe for treating PSD without the induction of mania (Robinson \& Starkstein, 1990). Less is known about the efficacy and side-effects of fluoxetine and other selective serotonin-reuptake inhibitors in the treatment of organic mood disorders, although Morris (1991) has recently reported recurrent orgasmic sexual experiences induced by fluoxetine in a patient with PSD.

Since post-stroke bipolar affective disorder has mainly been reported after subcortical righthemisphere lesions (Starkstein et al, 1991), it is possible that in our case fluoxetine may have precipitated a latent bipolar disorder. Therefore, further studies are needed to provide information on fluoxetine's safety profile in patients with PSD and righthemisphere damage.

Lebegue, B. (1987) Mania precipitated by fluoxetine (letter). American Journal of Psychiatry, 144, 1620.

MorRIs, P. L. (1991) Fluoxetine and orgasmic sexual experiences. International Journal of Psychiatry in Medicine, 21, 379-382.

Robinson, R. G. \& Starkstein, S. E. (1990) Current research

in affective disorders following stroke. The Journal of Neuropsychiatry and Clinical Neurosciences, 2, 1-14.

Settle, E. C. JR \& Setrle, G. P. (1984) A case of mania associated with fluoxetine. American Journal of Psychiatry, 141, 280-281. 
Starkstein, S. E., Fedoroff, P., Berthier, M. L., et al (1991) Manic-depressive and pure manic states after brain lesions. Biological Psychiatry, 29, 149-158.

\section{MARCELO L. BERTHIER \\ Virgen de la Victoria University Hospital Apartment 3091, 29010 Málaga, Spain}

Sant Pau Hospital

JAIME KULISEVSKY

Autonomous University of Barcelona, Spain

\section{Relapse following withdrawal of drug addiction}

SIR: There are few published follow-up studies from developing countries on relapse following withdrawal of opiate addiction. I wish to report part of a follow-up study which was carried out on detoxified patients discharged from the Post Graduate Hospital, Dhaka, Bangladesh. The pattern of relapse, effect of group therapy, and probable variables that predict relapse were examined.

Case report. We studied 50 subjects consecutively admitted to the psychiatric unit from December 1987 to July 1988 for detoxification. All were men with a mean age of 28.6 years. In $77 \%$ of cases heroin was smoked. Treatment included a two-week withdrawal period with symptomatic medication (chlorpromazine and benzodiazepines) followed by outpatient group therapy for six months. Data were obtained through a questionnaire interview schedule. We measured variables such as peer pressure, causes of relapse stated by patient, family and work situation, type and amount of drugs used in relapse, and physical and sexual difficulties after discharge on a five-point scale.

Subjects were interviewed on admission to the unit before detoxification had begun. The interview covered sociodemographic features including details of drug history. Further interviews were made on the first, third and sixth month following discharge. An operational definition of relapse and abstinence was formulated for the project. We considered relapse as a single use of addiction-forming drugs during follow-up (Hall et al, 1991). Abstinence was defined as complete absence of any addictive drug during the period. Only 12 patients voluntarily attended follow-up interviews; information about the rest were obtained by home visits.

By the end of the sixth month, seven subjects remained abstinent. Twenty-eight subjects remained drug free until the one-month follow-up point. At the end of the third month this figure was reduced to less than half (11).

A recent report of outcome by Gossop et al (1987) is rather optimistic. Our high relapse rate has to be weighed against the absence of any rehabilitation package. Most patients used less amounts of the addictive drug at relapse than before treatment.

We were interested in the causes of relapse as stated by the subjects. Our results show evidence of peer pressure in $15(34.88 \%)$ of 43 relapses in the form of constant exposure to drug-related cues and offering of drugs (Marlatt \& Gordon, 1985). Craving as a cause of relapse was mentioned by another 11 subjects.

Our results, both in terms of the high relapse rate and the psychological factors that may contribute to this, are strikingly similar to those reported from the West and illustrate the truly international nature of the future of opiate addiction.

Gossop, M., Green, L., PHILlips, G., et al (1987) What happens to opiate addicts immediately after treatment: a prospective study. British Medical Journal, 294, 1377-1380.

Hall, S. M., Wasserman, D. A. \& Havassy, B. E. (1991) Relapse prevention. NIDA Research Monograph, 106, 279-292.

Marlatt, G. A. \& Gordon, J. R. (1985) Relapse Prevention. New York: Guilford Press.

Institute of Psychiatry

Rezaul IsLam

De Crespigny Park

Denmark Hill

London SE5 8AF

\section{Percutaneous endoscopic gastrostomy and severe} endogenous depression

SIR: Percutaneous endoscopic gastrostomy (PEG) is a method for obtaining access to the stomach in patients requiring long-term tube-feeding (Bussone et al, 1992). The tube is inserted using a gastroscope while the patient is sedated. Since its first description, PEG has become one of the favoured techniques of feeding patients with persistent swallowing difficulty due to neurological or oropharyngeal disorders (Gauderer et al, 1980). We recently used this method of feeding in a patient who presented with severe endogenous depression with secondary anorexia and weight loss who improved markedly after electroconvulsive therapy (ECT) after stabilisation of her nutritional status with enteric feeding via PEG.

Case report. A 56-year-old woman was admitted from her home to the psychiatric hospital under Section 2 of the Mental Health Act 1983 with a diagnosis of severe endogenous depression. There was a recent concern by the community psychiatric nurse (CPN) over her poor and intermittent intake of foods and fluids. She had irrational ideas about her digestive processes and her bowels, and nihilistic delusions of what happened to food once she swallowed it. She had lost $19 \mathrm{~kg}$ in weight (her body weight was now $32 \mathrm{~kg}$ ) and her physical state on admission was of marked cachexia and dehydration with a urea concentration of $13.6 \mathrm{mmols} / \mathrm{l}$.

The intensity of her delusion made it impossible to obtain informed consent. A second opinion was obtained from an independent psychiatrist who authorised a course of ECT. Rehydration was started intravenously but her nutritional status continued to decline and she was referred for consideration of parenteral nutrition. It was decided that PEG would be a safer and more effective method of feeding. The 